Article

\title{
An $\alpha$-Monotone Generalized Log-Moyal Distribution with Applications to Environmental Data
}

\author{
Talha Arslan
}

Citation: Arslan, T. An $\alpha$-Monotone Generalized Log-Moyal Distribution with Applications to Environmental Data. Mathematics 2021, 9, 1400. https://doi.org/10.3390/math9121400

Academic Editors: Carlos Agra Coelho and Tatjana von Rosen

Received: 11 May 2021

Accepted: 14 June 2021

Published: 17 June 2021

Publisher's Note: MDPI stays neutral with regard to jurisdictional claims in published maps and institutional affiliations.

Copyright: (C) 2021 by the author. Licensee MDPI, Basel, Switzerland. This article is an open access article distributed under the terms and conditions of the Creative Commons Attribution (CC BY) license (https:// creativecommons.org/licenses/by/ $4.0 /)$.
Department of Econometrics, Van Yüzüncü Yil University, Van 65080, Turkey; mstalhaarslan@yyu.edu.tr

\begin{abstract}
Modeling environmental data plays a crucial role in explaining environmental phenomena. In some cases, well-known distributions, e.g., Weibull, inverse Weibull, and Gumbel distributions, cannot model environmental events adequately. Therefore, many authors tried to find new statistical distributions to represent environmental phenomena more accurately. In this paper, an $\alpha$-monotone generalized log-Moyal $(\alpha-\mathrm{G} \log M)$ distribution is introduced and some statistical properties such as cumulative distribution function, hazard rate function (hrf), scale-mixture representation, and moments are derived. The hrf of the $\alpha$-GlogM distribution can form a variety of shapes including the bathtub shape. The $\alpha$-GlogM distribution converges to generalized half-normal (GHN) and inverse GHN distributions. It reduces to slash GHN and $\alpha$-monotone inverse GHN distributions for certain parameter settings. Environmental data sets are used to show implementations of the $\alpha$-GlogM distribution and also to compare its modeling performance with its rivals. The comparisons are carried out using well-known information criteria and goodness-of-fit statistics. The comparison results show that the $\alpha$-GlogM distribution is preferable over its rivals in terms of the modeling capability.
\end{abstract}

Keywords: $\alpha$-monotone distribution; environmental data modeling; scale-mixture extension; slash distribution

\section{Introduction}

Modeling environmental data plays a crucial role in explaining environmental phenomena. In this context, there exists a much different statistical distribution, e.g., Weibull, inverse Weibull, and Gumbel distributions, to model the environmental data. However, in some cases, these distributions cannot model environmental events adequately. Therefore, many authors tried to find new statistical distributions to analyze them more accurately. For example, Gómez et al. [1] obtained a general family of skew-symmetric distributions generated by the cumulative distribution function of the normal distribution. They used skew Student- $t$-normal distribution to analyze nickel concentration in soil samples. Leiva et el. [2], Nadarajah [3], and Martinez et al. [4] proposed generalized BirnbaumSaunders, truncated inverted beta, and log-power-normal distributions, respectively, to model the air pollution data. Bakouch et al. [5] used a binomial-exponential 2 (BE2) distribution to analyze rainfall data. Asgharzadeh et al. [6] introduced the generalized BE2 distribution for the characterization of hydrological events. Gómez et al. [7] derived the slash Gumbel (SG) distribution and used it in modeling wind speed and snow accumulation data.

In recent years, many different statistical distributions have been introduced for modeling purposes. As stated in Bahti and Ravi's work [8], these distributions are usually obtained via well-known and widely used generalization or extension methods based on a transformation of the random variables, composition, compounding, and finite mixture of the distributions-see also Lee et al.'s work [9] for an overview on this context. Note that the most of distribution extension/generalization methods are based on the idea of adding a new parameter to the existing/baseline distribution; therefore, the procedure results in a much more flexible distribution than the existing one. In determining a baseline 
distribution, the attention is given to the distributions having a lower number of parameters while providing flexibility for modeling purposes. In this context, the Moyal distribution, introduced by Moyal in 1955, has drawn the attention of statisticians in recent years, and it has been widely used in physics for many years. It was derived as an explicit expression of Laondau's distribution - see Equation 4.6 in Moyal [10]. As stated in the work of Walck [11], a Moyal distribution can be defined as a universal form of energy loss by ionization for a fast charged particle and the number of ion pairs produced in this process.

In the literature, there are papers that include extensions/generalizations of the Moyal distribution. For example, Cordeiro [12] proposed a beta Moyal distribution and Genc et al. [13] achieved the beta Moyal slash distribution. Lastly, Bahti and Ravi [8] introduced a generalized form of the log-Moyal (GlogM) distribution. However, the Moyal distribution and its extensions/generalizations have been studied by the limited number of studies in the context of statistics. Therefore, in this study, an $\alpha$-monotone extension of the GlogM $(\alpha-G \log M)$ distribution is introduced to fill this gap in the corresponding literature. The $\alpha$-GlogM distribution is obtained as a product of independent $\operatorname{Glog} M(\beta, \sigma)$ and $(1 / \alpha)$ power of a Uniform on $(0,1)$, i.e., $\mathrm{U}(0,1)$, random variates. Hence, the resulting distribution has a wider range for the skewness and kurtosis values than the GlogM distribution.

This study has the following significant contributions. The $\alpha$-GlogM distribution is introduced. The $\alpha$-GlogM distribution reduces to $\alpha$-monotone inverse generalized halfnormal $(\alpha$-invGHN) and $\alpha$-monotone inverse half-normal $(\alpha$-invHN) distributions for the specific parameter settings. To the best of the author's knowledge, the $\alpha$-invGHN and $\alpha$-invHN distributions have not been introduced yet. The $\alpha$-GlogM distribution also converges to inverse generalized half-normal (invGHN) and inverse half-normal (invHN) distributions as limiting distributions. The $\alpha-G \log M$ distribution becomes the slash halfnormal (SHN) distribution proposed by Olmos et al. [14] and slash generalized half-normal (SGHN) distribution introduced by Olmos et al. [15] under the particular transformation of a random variable and parameters settings.

The rest of the paper is organized as follows. Section 2 presents the background information. The $\alpha$-GlogM distribution and its properties are presented in Section 3. Maximum likelihood (ML) and method of moments (MoM) estimations of the parameters of the $\alpha$-GlogM distribution and Monto-Carlo simulation results for them are given in Section 4. Section 5 includes applications with environmental data sets for illustrating the implementation of the $\alpha$-GlogM distribution. The paper finishes with some concluding remarks.

\section{Background Information}

In this section, some brief background information is provided to follow the rest of the paper easily. The following subsections present the GlogM distribution and some of its properties, as well as concise information for the slash and $\alpha$-monotone distributions. Note that this study is constructed around these two subsections.

\subsection{The GlogM Distribution}

The GlogM distribution introduced by Bahti and Ravi [8] has the following probability density function (pdf):

$$
f_{X}(x ; \beta, \sigma)=\frac{1}{\sqrt{2 \pi} \beta} \sigma^{\frac{1}{2 \beta}} x^{-\left(1+\frac{1}{2 \beta}\right)} \exp \left(-\frac{1}{2} \sigma^{\frac{1}{\beta}} x^{-\frac{1}{\beta}}\right) ; \quad x>0, \quad \beta>0, \quad \sigma>0
$$

and cumulative distribution function (cdf)

$$
F_{X}(x ; \beta, \sigma)=\Gamma\left[0.5\left(\frac{\sigma}{x}\right)^{\frac{1}{\beta}}, 0.5\right] \text {. }
$$


Here, $\beta$ is the shape parameter, $\sigma$ is the scale parameter, and $\Gamma\left[0.5\left(\frac{\sigma}{x}\right)^{\frac{1}{\beta}}, 0.5\right]$ represents the upper-incomplete gamma function defined as

$$
\frac{1}{\Gamma(0.5)} \int_{0.5\left(\frac{\sigma}{x}\right)^{\frac{1}{\beta}}}^{\infty} u^{0.5-1} \exp (-u) d u
$$

Some distributional properties and actuarial measures of the GlogM distribution were derived by Bahti and Ravi [8]. Hereinafter, the random variable $X$, having the pdf given in (1), will be represented as $X \sim \operatorname{Glog} M(\beta, \sigma)$.

The GlogM distribution is reduced to the families of distributions given in the following propositions.

Proposition 1. Let $X \sim \operatorname{Glog} M(\beta, \sigma)$.

i. If $\beta=1 /(2 \lambda)$ and $\sigma=1 / \theta$, the $p d f$ of the random variable $X$ reduces to the inv $G H N$ distribution

$$
f_{X}(x ; \beta=1 /(2 \lambda), \sigma=1 / \theta)=\sqrt{\frac{2}{\pi}} \frac{\lambda}{\theta^{\lambda}} x^{-(\lambda+1)} \exp \left(-\frac{1}{2}\left(\frac{1}{\theta x}\right)^{2 \lambda}\right) .
$$

ii. If $\beta=1 / 2$ and $\sigma=1 / \theta$, the $p d f$ of the random variable $X$ reduces to the inv $H N$ distribution

$$
f_{X}(x ; \beta=1 / 2, \sigma=1 / \theta)=\sqrt{\frac{2}{\pi}} \frac{1}{\theta} x^{-2} \exp \left(-\frac{1}{2}\left(\frac{1}{\theta x}\right)^{2}\right) .
$$

Proposition 2. Let $Z=X^{-1}$, then it has the pdf

$$
f_{Z}(z ; \beta, \sigma)=\frac{1}{\sqrt{2 \pi} \beta} \sigma^{\frac{1}{2 \beta}} z^{\left(\frac{1}{2 \beta}-1\right)} \exp \left(-\frac{1}{2}(\sigma z)^{\frac{1}{\beta}}\right) ; \quad z>0, \quad \beta>0, \quad \sigma>0 .
$$

Then:

i. If $\beta=1 /(2 \lambda)$ and $\sigma=1 / \theta$, the $p d f$ of the random variable $Z$ reduces to the generalized half-normal (GHN) distribution proposed by Cooray and Ananda [16];

ii. If $\beta=1 / 2$ and $\sigma=1 / \theta$, the pdf of the random variable $Z$ reduces to the half-normal (HN) distribution.

See Bahti and Ravi's research [8] for further details.

\subsection{The Slash and $\alpha$-Monotone Distributions}

The slash distribution has drawn attention from the practitioners, since Andrews et al. [17] introduced the slash distribution as the distribution of $Z / Y$, where $Z$ and $Y$ are independent random variables following the standard-normal and $U(0,1)$ distributions, respectively. This distribution is also called the canonical slash distribution and has heavier tails than a standard-normal distribution. Therefore, it plays an essential role in robustness studiessee Rogers and Tukey [18] and Mosteller and Tukey [19].

Later on, various distributions are introduced based on the same philosophy of the Andrews et al. [17]. They are also called slash distributions and are obtained by replacing the nominator with some well-known distribution and the denominator with the $(1 / \alpha)$ power of $\mathrm{U}(0,1)$. Actually, Jones [20] called such distributions $\alpha$-slash distributions.

In the literature, interesting studies on slash distributions are available considering both theoretical and practical viewpoints. For example, see Gómez et al. [21], Genc [22], Punathumparambath [23], Olmos et al. [15], Astorga et al. [24], Korkmaz [25], Gómez et al. [7] and references given in these studies for the univariate slash distribution. Additionally, see, for example, Arslan [26] and Arslan and Genc [27] in the context of multivariate slash distributions.

Slash distributions have a simple concept; therefore, this leads to researchers introducing useful modified/generalized/extended slash distributions. These distributions differ 
from the usual slash distributions by replacing a denominator with an exponential, specific gamma or beta distributions-see Reyes et al. [28], Iriarte et al. [29], Reyes et al. [30], and Rojas et al. [31], respectively. See also Zörnig [32] for generalized slash distributions with representation by hypergeometric functions.

Recently, Jones [20] conducted a study on univariate slash distributions, for both continuous and discrete cases. In Appendix-A of this paper, Jones [20] also considered the distribution of a form with multiplication signs rather than the division signs. Jones [20] called it the $\alpha$-monotone distribution. See Arslan [33] for an example in the context of $\alpha$-monotone inverse Weibull distributions.

The basic theory and conditions of the slash and $\alpha$-monotone distributions are not given here for the sake of brevity. Here, I refer to Jones' work [20] and references therein for detailed information about theoretical viewpoints.

\section{The $\alpha$-GlogM Distribution}

In this section, the $\alpha$-GlogM distribution is introduced by using the stochastic representation of the $\alpha$-monotone distribution. Some statistical properties of the $\alpha$-GlogM are also provided.

\subsection{Density Function and Some Statistical Properties}

Definition 1. Random variable $T$ defined by the stochastic representation

$$
T=X \times Y^{1 / \alpha}, \quad \alpha>0
$$

has the $\alpha-G \log M$ distribution, denoted as $T \sim \alpha-G \log M(\alpha, \beta, \sigma)$, where independent random variables $X$ and $Y$ follow the $\operatorname{Glog} M(\beta, \sigma)$ and $U(0,1)$ distributions, respectively.

Proposition 3. Pdf of the random variable Thaving the $\alpha-G \log M$ distribution is

$$
f_{T}(t ; \alpha, \beta, \sigma)=\frac{\alpha 2^{\alpha \beta}}{\sigma^{\alpha} \sqrt{\pi}} \Gamma(\alpha \beta+0.5) t^{\alpha-1} G\left(t^{-\frac{1}{\beta}}, \alpha \beta+0.5,0.5 \sigma^{\frac{1}{\beta}}\right)
$$

where $\alpha$ and $\beta$ are the shape parameters, $\sigma$ is the scale parameter, and $\Gamma(\cdot)$ represents the gamma function. Here,

$$
G(t, a, b)=\frac{b^{a}}{\Gamma(a)} \int_{0}^{t} u^{a-1} \exp (-b u) d u
$$

is the cdf of the Gamma distribution with shape parameter a and scale parameter $b$.

Proof. The proof is completed by using the stochastic representation in (3) and the Jacobian transformation as follows:

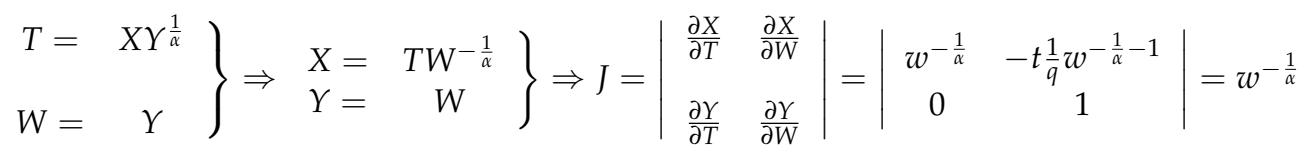

where $J$ is the Jacobian transformation. Then, the joint pdf of $T$ and $W$ is

$$
\begin{aligned}
f_{T, W}(t, w) & =f_{X, Y}(x(t, w), y(t, w))|J| \\
& =\frac{\sigma^{\frac{1}{2 \beta}}}{\beta \sqrt{2 \pi}} w^{-\frac{1}{\alpha}}\left(t w^{-\frac{1}{\alpha}}\right)^{-\left(1+\frac{1}{\beta}\right)} \exp \left(-0.5 \sigma^{\frac{1}{\beta}}\left(t w^{-\frac{1}{\alpha}}\right)^{-\frac{1}{\beta}}\right) .
\end{aligned}
$$

The marginal pdf of the random variable $T$ in (4) is obtained immediately by taking integration with respect to the random variable $W$ using the transformation $t^{-\frac{1}{\beta}} w^{\frac{1}{\alpha \beta}}=$ $u$. 
Proposition 4. The distribution of random variable T, defined in (3), is an $\alpha$-monotone GlogM distribution, since $T$ has an $\alpha$-monotone density iff

$$
\frac{d}{d t}\left(\log f_{T}\right) \leq \frac{\alpha-1}{t}, \text { for all } t>0
$$

Proof. From Proposition 3,

$$
f_{T}(t ; \alpha, \beta, \sigma)=\int_{0}^{1} \frac{\sigma^{\frac{1}{2 \beta}}}{\beta \sqrt{2 \pi}} w^{-\frac{1}{\alpha}}\left(t w^{-\frac{1}{\alpha}}\right)^{-\left(1+\frac{1}{\beta}\right)} \exp \left(-0.5 \sigma^{\frac{1}{\beta}}\left(t w^{-\frac{1}{\alpha}}\right)^{-\frac{1}{\beta}}\right) d w .
$$

By using the variable transformation $t w^{-1 / \alpha}=u, f_{T}(t)$ is expressed as

$$
\begin{aligned}
f_{T}(t ; \alpha, \beta, \sigma) & =\int_{t}^{\infty} \alpha t^{\alpha-1} \frac{\sigma^{\frac{1}{2 \beta}}}{\beta \sqrt{2 \pi}} u^{-\alpha} u^{-\left(1+\frac{1}{\beta}\right)} \exp \left(-0.5 \sigma^{\frac{1}{\beta}} u^{-\frac{1}{\beta}}\right) d u \\
& =\alpha t^{\alpha-1} \int_{t}^{\infty} \frac{1}{u^{\alpha}} f_{X}(u ; \beta, \sigma) d u .
\end{aligned}
$$

It is seen that $f_{T}(t)$ satisfies

$$
f_{T}(t)=\alpha t^{\alpha-1} \int_{t}^{\infty} \frac{1}{x^{\alpha}} f_{X}(x) d x .
$$

Then,

$$
\begin{aligned}
f_{T}^{\prime}(t) & =\alpha(\alpha-1) t^{\alpha-2} \int_{t}^{\infty} \frac{1}{x^{\alpha}} f_{X}(x) d x-\left(\alpha t^{\alpha-1}\right) \frac{1}{t^{\alpha}} f_{X}(t) \\
& =(\alpha-1) t^{-1} f_{T}(t)-\alpha t^{-1} f_{X}(t) \\
\alpha f_{X}(t) & =(\alpha-1) f_{T}(t)-t f_{T}^{\prime}(t) .
\end{aligned}
$$

From there

$$
\begin{aligned}
(\alpha-1) f_{T}(t)-t f_{T}^{\prime}(t) & \geq 0 \quad \text { since } \quad f_{X}(t) \geq 0 \\
\frac{\alpha-1}{t} & \geq \frac{f_{T}^{\prime}(t)}{f_{T}(t)} \\
\frac{\alpha-1}{t} & \geq \frac{d}{d t} \log \left(f_{T}(t)\right) \\
\frac{d}{d t} \log \left(f_{T}(t)\right) & \leq \frac{\alpha-1}{t}
\end{aligned}
$$

The proof is completed; see Appendix-A in Jones [20] for further details about the $\alpha$-monotone density.

Proposition 5. If $T \mid Y=y \sim G \log M\left(\beta, \sigma y^{\frac{1}{\alpha}}\right)$ and $Y \sim U(0,1)$, then $T \sim \alpha-G \log M(\alpha, \beta, \sigma)$.

Proof.

$$
\begin{aligned}
f_{T}(t ; \alpha, \beta, \sigma) & =\int_{0}^{1} f_{X}\left(t ; \beta, \sigma y^{\frac{1}{\alpha}}\right) f_{Y}(y) d y \\
& =\frac{1}{\beta \sqrt{2 \pi}} \int_{0}^{1}\left(\sigma y^{\frac{1}{\alpha}}\right)^{\frac{1}{2 \beta}} t^{-\left(1+\frac{1}{2 \beta}\right)} \exp \left[-0.5\left(\sigma y^{\frac{1}{\alpha}}\right)^{\frac{1}{\beta}} t^{-\frac{1}{\beta}}\right] d y
\end{aligned}
$$

The proof is completed immediately after the following transformation, $t^{-\frac{1}{\beta}} y^{\frac{1}{\alpha \beta}}=$ $u$. 
Remark 1. From Proposition 5, it is clear that the $\alpha-\operatorname{Glog} M(\alpha, \beta, \sigma)$ distribution is a scale-mixture between the $\operatorname{Glog} M\left(\beta, \sigma y^{\frac{1}{\alpha}}\right)$ and $U(0,1)$ distributions.

Proposition 6. Let $T \sim \alpha-G \log M(\alpha, \beta, \sigma)$. Then,

i. $\quad Z=a T \sim \alpha-G \log M(\alpha, \beta, a \sigma)$.

ii. The pdf of $Z=T^{-1}$ is

$$
f_{Z}(z ; \alpha, \beta, \sigma)=\frac{\alpha 2^{\alpha \beta}}{\sigma^{\alpha} \sqrt{\pi}} \Gamma(\alpha \beta+0.5) t^{-(\alpha+1)} G\left(t^{\frac{1}{\beta}}, \alpha \beta+0.5,0.5 \sigma^{\frac{1}{\beta}}\right), \quad z>0 .
$$

iii. The pdf of $Z=\ln T$ is

$$
f_{Z}(z ; \alpha, \beta, \sigma)=\frac{\alpha 2^{\alpha \beta}}{\sigma^{\alpha} \sqrt{\pi}} \Gamma(\alpha \beta+0.5) \exp (\alpha z) G\left(\exp \left(-\frac{z}{\beta}\right), \alpha \beta+0.5,0.5 \sigma^{\frac{1}{\beta}}\right), \quad z \in \mathbb{R} .
$$

Proof. The results follow the change-of-variable technique.

Remark 2. The first part of Proposition 6 shows that $\alpha$-Glog $M$ distributions belong to the scale family. Therefore, if $T \sim \alpha-G \log M(\alpha, \beta, \sigma)$, then $\frac{1}{\sigma} T \sim \alpha-G \log M(\alpha, \beta, 1)$. The random variable $Z$ with a pdf given in the second part of Proposition 6 follows the SGHN distribution proposed by Olmos et al. [15] with a certain reparameterization. The result in the third part of the Proposition 6 can be used to study the regression model for positive random variables. See Iriarte [34] for an example in the context of slashed generalized Rayleigh (SGR) distributions and references therein for the regression model for the positive random variables.

Proposition 7. The cdf of the $\alpha-G \log M$ distribution is

$$
\begin{aligned}
F_{T}(t ; \alpha, \beta, \sigma) & =F_{X}(t ; \beta, \sigma)+\frac{t}{\alpha} f_{T}(t ; \alpha, \beta, \sigma) \\
& =\Gamma\left[0.5\left(\frac{\sigma}{t}\right)^{\frac{1}{\beta}}, 0.5\right]+\frac{2^{\alpha \beta}}{\sigma^{\alpha} \sqrt{\pi}} \Gamma(\alpha \beta+0.5) t^{\alpha} G\left(t^{-\frac{1}{\beta}}, \alpha \beta+0.5,0.5 \sigma^{\frac{1}{\beta}}\right) .
\end{aligned}
$$

Proof. The result follows from the definition of the $\alpha$-monotone distribution-see AppendixA in Jones' work [20].

Proposition 8. Hazard rate function ( $h r f$ ) of the random variable $T$ with the $\alpha-G \log M$ distribution is

$$
h_{T}(t ; \alpha, \beta, \sigma)=\frac{\frac{\alpha 2^{\alpha \beta}}{\sigma^{\alpha} \sqrt{\pi}} \Gamma(\alpha \beta+0.5) t^{\alpha-1} G\left(t^{-\frac{1}{\beta}}, \alpha \beta+0.5,0.5 \sigma^{\frac{1}{\beta}}\right)}{1-\Gamma\left[0.5\left(\frac{\sigma}{t}\right)^{\frac{1}{\beta}}, 0.5\right]-\frac{2^{\alpha \beta}}{\sigma^{\alpha} \sqrt{\pi}} \Gamma(\alpha \beta+0.5) t^{\alpha} G\left(t^{-\frac{1}{\beta}}, \alpha \beta+0.5,0.5 \sigma^{\frac{1}{\beta}}\right)} .
$$

Proof. The result follows from the definition of the hrf function.

In Figure 1, different density plots of the $\alpha$-GlogM distribution are illustrated for certain values of the distribution parameters. The $\alpha-G \log M$ distribution can be skewed left or right based on the different parameter settings-see Figure 1a. Its pdf also has a triangular or rectangular shape-see Figure $1 \mathrm{~b}$. It can be concluded that the shape parameter $\alpha$ plays an important role in controlling the kurtosis of the distribution (see Figure 1a) and changing the shape of the pdf of the distribution (see Figure 1b).

Note that the hrf of the $\alpha$-GlogM distribution can be expressed by using the Propositions 3 and 7 in terms of the upper-incomplete gamma function and cdf of a gamma distribution. However, analyzing the mathematical properties of the hrf of the $\alpha$-GlogM distribution is 
intractable. Therefore, in Figure 1c, hrf of the $\alpha$-GlogM distribution is plotted for the certain values of the parameters to show the different shapes of it.

Figure 1c shows that for the particular values of the parameters, the hrf of the $\alpha$-GlogM distribution can form a variety of shapes such as monotonically decreasing, monotonically increase-decrease and bathtub(monotonically decrease-increase-decrease) shapes.

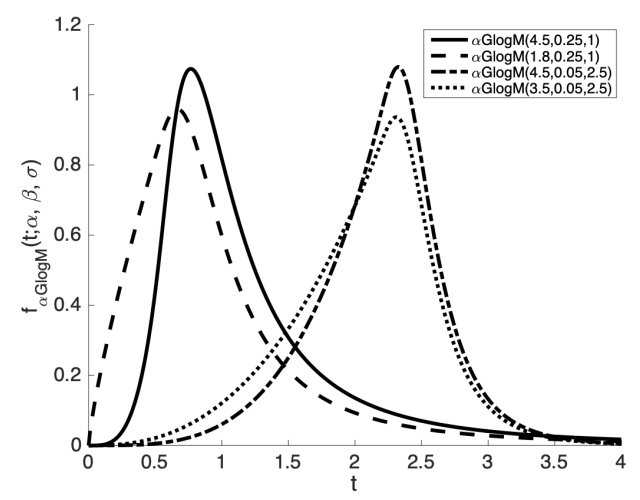

(a)

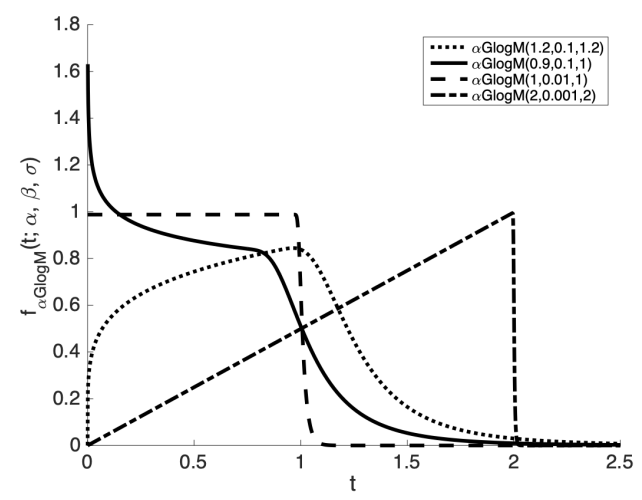

(b)

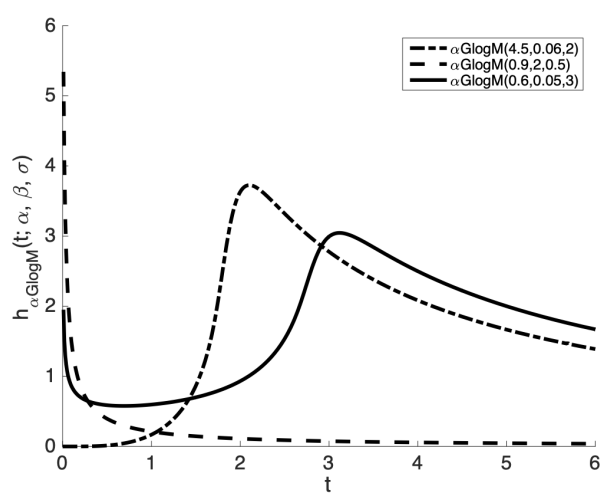

(c)

Figure 1. The density plots, $(\mathbf{a}, \mathbf{b})$ and hrfs (c) of the $\alpha$-GlogM distribution for certain values of the distribution parameters.

\subsection{Moments}

The moments of the random variable having the $\alpha$-GlogM distribution were obtained immediately via the stochastic representation given in (3). Following lemma is utilized to acquire the moments of the random variable with the $\alpha$-GlogM distribution.

Lemma 1. Let $X \sim \operatorname{Glog} M(\beta, \sigma)$ and $Y \sim U(0,1)$ be independent random variables; then, the $r$-th moment of $X$ and the $(r / \alpha)$-th moment of the $Y$ are

$$
\mathbb{E}\left[X^{r}\right]=\frac{\sigma^{r}}{2^{r \beta} \Gamma(0.5)} \Gamma(0.5-r \beta), \quad r \beta<0.5 \quad \text { and } \quad \mathbb{E}\left[Y^{\frac{r}{\alpha}}\right]=\frac{\alpha}{\alpha+r^{\prime}},
$$

respectively.

Proposition 9. Let $T \sim \alpha-G \log M(\alpha, \beta, \sigma)$; then, the $r$-th moment of the $\alpha-G \log M$ distribution is formulated by

$$
\mathbb{E}\left[T^{r}\right]=\frac{\sigma^{r}}{\sqrt{\pi} 2^{r \beta}} \Gamma(0.5-r \beta) \frac{\alpha}{\alpha+r}, \quad r \beta<0.5 .
$$


Proof. By using the stochastic representation in (3), we obtain

$$
\mathbb{E}\left[T^{r}\right]=\mathbb{E}\left[X^{r}\right] \mathbb{E}\left[Y^{\frac{r}{\alpha}}\right]
$$

in which the expectations $\mathbb{E}\left[X^{r}\right]$ and $\mathbb{E}\left[Y^{\frac{r}{\alpha}}\right]$ have been obtained in Lemma 1.

Corollary 1. The mean and variance of the random variable $T$ having the $\alpha-G \log M(\alpha, \beta, \sigma)$ distribution are

$$
\mathbb{E}(T)=\frac{\alpha \sigma}{\sqrt{\pi} 2^{\beta}(\alpha+1)} \Gamma(0.5-\beta) ; \quad \beta<0.5
$$

and

$$
\mathbb{V}(T)=\frac{\alpha \sigma^{2}\left[\sqrt{\pi}(\alpha+1)^{2} \Gamma(0.5-2 \beta)-\alpha(\alpha+2) \Gamma^{2}(0.5-\beta)\right]}{\pi 4^{\beta}(\alpha+1)^{2}(\alpha+2)} ; \quad \beta<0.25,
$$

respectively.

Corollary 2. The skewness $\left(\sqrt{\beta_{1}}\right)$ and kurtosis $\left(\beta_{2}\right)$ coefficients of the $\alpha-G \log M(\alpha, \beta, \sigma)$ distribution can be obtained by using (5) via the software MATHEMATICA. They are not given here for the sake of brevity. However, the surface plots of the $\sqrt{\beta_{1}}$ and $\beta_{2}$ measures of the $\alpha$-GlogM distribution are illustrated in Figure 2.

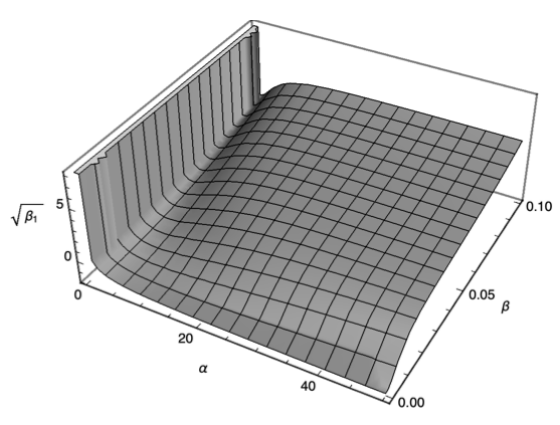

(a) Skewness

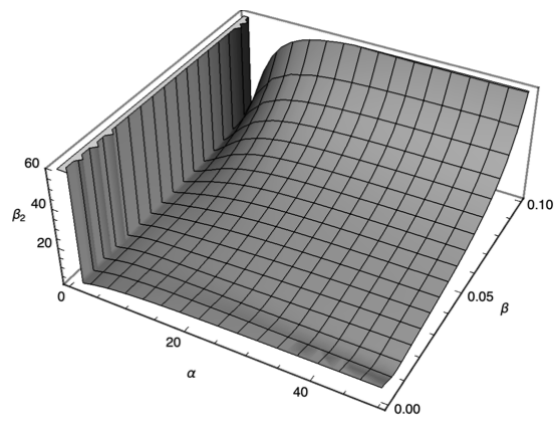

(b) Kurtosis

Figure 2. The surface plots of the skewness and kurtosis measures of the $\alpha$-GlogM distribution for $0<\beta<0.1$ and $0<\alpha<50$.

\subsection{Related Distributions}

In this subsection, submodels of the $\alpha$-GlogM distribution and also limiting distributions are provided.

\subsubsection{Submodels}

Let random variable $T$ follow the $\alpha-\operatorname{Glog} \mathrm{M}(\alpha, \beta, \sigma)$, having the pdf given in (4).

i. If $\beta=1 /(2 \lambda)$ and $\sigma=1 / \theta$, then $T$ has $\alpha$-invGHN density

$$
f_{T}(t ; \alpha, \lambda, \theta)=\alpha \theta^{\alpha} \sqrt{\frac{2^{\alpha / \lambda}}{\pi}} \Gamma\left(\frac{\alpha+\lambda}{2 \lambda}\right) t^{\alpha-1} G\left[\left(\frac{1}{t}\right)^{2 \lambda}, \frac{\alpha+\lambda}{2 \lambda}, \frac{1}{2 \theta^{2 \lambda}}\right] ; \quad t>0, \quad \alpha, \lambda, \theta>0 .
$$

ii. If $\beta=1 / 2$ and $\sigma=1 / \theta$, then $T$ has $\alpha$-invHN density

$$
f_{T}(t ; \alpha, \theta)=\alpha \theta^{\alpha} \sqrt{\frac{2^{\alpha}}{\pi}} \Gamma\left(\frac{\alpha+1}{2}\right) t^{\alpha-1} G\left[\left(\frac{1}{t}\right)^{2}, \frac{\alpha+1}{2}, \frac{1}{2 \theta^{2}}\right] ; \quad t>0, \quad \alpha, \theta>0 .
$$


iii. If $\beta=1 /(2 \lambda)$ and $\sigma=1 / \theta$, then a random variable $Z=T^{-1}$ follows the SGHN distribution proposed by Olmos et al. [15] with pdf

$$
g_{Z}(z ; \alpha, \lambda, \theta)=\alpha \theta^{\alpha} \sqrt{\frac{2^{\alpha / \lambda}}{\pi}} \Gamma\left(\frac{\alpha+\lambda}{2 \lambda}\right) t^{-(\alpha+1)} G\left[z^{2 \lambda}, \frac{\alpha+\lambda}{2 \lambda}, \frac{1}{2 \theta^{2 \lambda}}\right] ; \quad t>0, \quad \alpha, \lambda, \theta>0 .
$$

iv. If $\beta=1 / 2$ and $\sigma=1 / \theta$, then a random variable $Z=T^{-1}$ follows the SHN distribution proposed by Olmos et al. [14] with pdf

$$
g_{Z}(z ; \alpha, \theta)=\alpha \theta^{\alpha} \sqrt{\frac{2^{\alpha}}{\pi}} \Gamma\left(\frac{\alpha+1}{2}\right) t^{-(\alpha+1)} G\left[z^{2}, \frac{\alpha+1}{2}, \frac{1}{2 \theta^{2}}\right] ; \quad t>0, \quad \alpha, \theta>0 .
$$

\subsubsection{Limiting Distributions}

Let the $\alpha$-GlogM distribution have the pdf given in (4).

i. If $\alpha \rightarrow \infty$, then the $\alpha-\operatorname{GlogM}(\alpha, \beta, \sigma)$ converges to the $\operatorname{GlogM}(\beta, \sigma)$ distribution given in (1), i.e.,

$$
\lim _{\alpha \rightarrow \infty} f_{T}(t ; \alpha, \beta, \sigma)=f_{X}(t ; \beta, \sigma)
$$

ii. If $\alpha \rightarrow \infty$, and $\beta=1 /(2 \lambda)$ and $\sigma=1 / \theta$, then the $\alpha-\operatorname{GlogM}(\alpha, \lambda, \theta)$ converges to the invGHN distribution.

$$
g_{T}(t ; \lambda, \theta)=\sqrt{\frac{2}{\pi}} \frac{\lambda}{\theta^{\lambda}} t^{-(\lambda+1)} \exp \left[-\frac{1}{2}\left(\frac{1}{\theta x}\right)^{2 \lambda}\right] ; \quad t>0, \quad \lambda, \theta>0 .
$$

iii. If $\alpha \rightarrow \infty$, and $\beta=1 / 2$ and $\sigma=1 / \theta$, then the $\alpha-\operatorname{GlogM}(\alpha, \theta)$ converges to the invHN distribution.

$$
g_{T}(t ; \theta)=\sqrt{\frac{2}{\pi}} \frac{1}{\theta} t^{-2} \exp \left[-\frac{1}{2}\left(\frac{1}{\theta x}\right)^{2}\right] ; \quad t>0, \quad \theta>0 .
$$

iv. Let $Z=T^{-1}$. If $\alpha \rightarrow \infty$ and $\beta=1 /(2 \lambda)$ and $\sigma=1 / \theta$, then the pdf of the random variable $Z$ converges to the GHN distribution proposed by Cooray and Ananda [16].

$$
g_{Z}(z ; \lambda, \theta)=\sqrt{\frac{2}{\pi}} \frac{\lambda}{\theta^{\lambda}} z^{\lambda-1} \exp \left[-\frac{1}{2}\left(\frac{z}{\theta}\right)^{2 \lambda}\right] ; \quad z>0, \quad \lambda, \theta>0 .
$$

v. Let $Z=T^{-1}$. If $\alpha \rightarrow \infty$, and $\beta=1 / 2$ and $\sigma=1 / \theta$, then the pdf of the random variable $Z$ converges to the $H N$ distribution.

$$
g_{Z}(z ; \theta)=\sqrt{\frac{2}{\pi}} \frac{1}{\theta} \exp \left[-\frac{1}{2}\left(\frac{z}{\theta}\right)^{2}\right] ; \quad t>0, \quad \theta>0 .
$$

\subsection{Data Generation}

The following steps are used in generating random variates from the $\alpha$-GlogM distribution in (4). Note that stochastic representation in (3) is used for the data generation process.

Step 1. Generate a $p$ from $\mathrm{U}(0,1)$ distribution and incorporate it into the equation

$$
x=\sigma \exp \left[-\beta \ln \left(2 \Gamma^{-1}(p, 0.5)\right)\right]
$$

to generate a random number $x$ from the $\operatorname{GlogM}(\beta, \sigma)$ distribution. Here, $\Gamma^{-1}(\cdot, \cdot)$ is inverse of the upper-incomplete gamma function.

Step 2. Generate a $y$ from a $\mathrm{U}(0,1)$ distribution and incorporate it into the equation

$$
t=x \times y^{1 / \alpha}
$$


to generate the random varaites from the $\alpha$-GlogM distribution.

Remark 3. Notice that the equality given in Step 1 is obtained by using the relation GlogM distribution with the Moyal distribution, i.e., if random variable $Z$ follows a Moyal distribution, then $X=\sigma \exp (\beta Z)$ has a $G \log M$ distribution. This representation does not alter the data generating process from the $\alpha-G \log M$ distribution. Alternatively, $x=\sigma\left[2 \Gamma^{-1}(p, 0.5)\right]^{-\beta}$ can be used to generate random variates from the $\operatorname{Glog} M(\alpha, \beta)$ distribution. This alternative equality is obtained by taking the inverse of the cdf of the GlogM distribution given in (2).

\section{Estimation}

In this section, the ML and MoM estimations of the parameters of the $\alpha$-GlogM distribution are provided. Then, a Monte-Carlo simulation experiment is carried out to compare the efficiencies of the ML estimators of the parameters $\alpha, \beta$ and $\sigma$ with their MoM counterparts in terms of the bias, variance and mean squared error (MSE) criteria.

\subsection{Estimation}

Let $t_{1}, t_{2}, \ldots, t_{n}$ be a random sample from the $\alpha$-GlogM distribution. The ML estimates of the parameters $\alpha, \beta$ and $\sigma$ are the points at which the $\log$-likelihood $(\ln L)$ function

$$
\begin{aligned}
\ln L= & -\frac{n}{2} \ln \pi+n \ln \alpha+n \alpha \beta \ln 2-n \alpha \ln \sigma+n \ln [\Gamma(\alpha \beta+0.5)] \\
& +(\alpha-1) \sum_{i=1}^{n} \ln t_{i}+\sum_{i=1}^{n} \ln \left[G\left(t_{i}^{-\frac{1}{\beta}}, \alpha \beta+0.5,0.5 \sigma^{\frac{1}{\beta}}\right)\right]
\end{aligned}
$$

attains its maximum. After taking partial derivatives of the $\ln L$ with respect to the parameters $\alpha, \beta$ and $\sigma$ and then setting them equal to 0 , likelihood equations

$$
\begin{aligned}
& \frac{\partial \ln L}{\partial \alpha}=\frac{n}{\alpha}+n \beta \ln 2-n \ln \sigma+n \beta \psi(\alpha \beta+0.5)+\sum_{i=1}^{n} \ln t_{i}+\sum_{i=1}^{n} \frac{\frac{d}{d \alpha} G\left(t_{i}^{-\frac{1}{\beta}}, \alpha \beta+0.5,0.5 \sigma^{\frac{1}{\beta}}\right)}{G\left(t_{i}^{-\frac{1}{\beta}}, \alpha \beta+0.5,0.5 \sigma^{\frac{1}{\beta}}\right)}=0, \\
& \frac{\partial \ln L}{\partial \beta}=n \alpha \ln 2+n \alpha \psi(\alpha \beta+0.5)+\sum_{i=1}^{n} \frac{\frac{d}{d \beta} G\left(t_{i}^{-\frac{1}{\beta}}, \alpha \beta+0.5,0.5 \sigma^{\frac{1}{\beta}}\right)}{G\left(t_{i}^{-\frac{1}{\beta}}, \alpha \beta+0.5,0.5 \sigma^{\frac{1}{\beta}}\right)}=0,
\end{aligned}
$$

and

$$
\frac{\partial \ln L}{\partial \sigma}=-\frac{n \alpha}{\sigma}+\sum_{i=1}^{n} \frac{\frac{d}{d \sigma} G\left(t_{i}^{-\frac{1}{\beta}}, \alpha \beta+0.5,0.5 \sigma^{\frac{1}{\beta}}\right)}{G\left(t_{i}^{-\frac{1}{\beta}}, \alpha \beta+0.5,0.5 \sigma^{\frac{1}{\beta}}\right)}=0
$$

are obtained. Here, $\psi(\cdot)$ denotes the digamma function-i.e., $\psi(x)=\frac{d}{d x} \ln \Gamma(x)$. It is clear that ML estimates of the unknown parameters of the $\alpha$-GlogM distribution, i.e., the values of the $\hat{\alpha}_{M L}, \hat{\beta}_{M L}$ and $\hat{\sigma}_{M L}$, can be obtained by using the numerical techniques such the as Newton-Raphson method. The observed information matrix, which is a symmetric matrix, i.e., $\mathbf{J}(\Theta)$ where $\Theta=(\alpha, \beta, \sigma)^{\top}$, is

$$
\mathbf{J}(\Theta)=\left[\begin{array}{lll}
\mathbf{J}_{\alpha \alpha} & \mathbf{J}_{\alpha \beta} & \mathbf{J}_{\alpha \sigma} \\
& \mathbf{J}_{\beta \beta} & \mathbf{J}_{\beta \sigma} \\
& & \mathbf{J}_{\sigma \sigma}
\end{array}\right] \text { where }
$$




$$
\begin{gathered}
\mathbf{J}_{\alpha \alpha}=\frac{n}{\alpha^{2}}-n \beta^{2} \psi_{1}(\alpha \beta+0.5)-\sum_{i=1}^{n} \frac{d}{d \alpha}\left[\frac{\frac{d}{d \alpha} G\left(t^{-\frac{1}{\beta}}, \alpha \beta+0.5,0.5 \sigma^{\frac{1}{\beta}}\right)}{G\left(t^{-\frac{1}{\beta}}, \alpha \beta+0.5,0.5 \sigma^{\frac{1}{\beta}}\right)}\right], \\
\mathbf{J}_{\beta \beta}=-n \alpha^{2} \psi_{1}(\alpha \beta+0.5)-\sum_{i=1}^{n} \frac{d}{d \beta}\left[\frac{\frac{d}{d \beta} G\left(t^{-\frac{1}{\beta}}, \alpha \beta+0.5,0.5 \sigma^{\frac{1}{\beta}}\right)}{G\left(t^{-\frac{1}{\beta}}, \alpha \beta+0.5,0.5 \sigma^{\frac{1}{\beta}}\right)}\right], \\
\mathbf{J}_{\alpha \sigma}=-\frac{n \alpha}{\sigma^{2}}-\sum_{i=1}^{n} \frac{d}{d \sigma}\left[\frac{\frac{d}{d \sigma} G\left(t^{-\frac{1}{\beta}}, \alpha \beta+0.5,0.5 \sigma^{\frac{1}{\beta}}\right)}{G\left(t^{-\frac{1}{\beta}}, \alpha \beta+0.5,0.5 \sigma^{\frac{1}{\beta}}\right)}\right], \\
\mathbf{J}_{\alpha \sigma=}=\frac{n}{\sigma}-\sum_{i=1}^{n} \frac{d}{d \sigma}\left[\frac{\frac{d}{d \alpha} G\left(t^{-\frac{1}{\beta}}, \alpha \beta+0.5,0.5 \sigma^{\frac{1}{\beta}}\right)}{G\left(t^{-\frac{1}{\beta}}, \alpha \beta+0.5,0.5 \sigma^{\frac{1}{\beta}}\right)}\right], \\
\mathbf{J}_{\beta \sigma}=-\sum_{i=1}^{n} \frac{d}{d \sigma}\left[\frac{\frac{d}{d \beta} G\left(t^{-\frac{1}{\beta}}, \alpha \beta+0.5,0.5 \sigma^{\frac{1}{\beta}}\right)}{G\left(t^{-\frac{1}{\beta}}, \alpha \beta+0.5,0.5 \sigma^{\frac{1}{\beta}}\right)}\right]
\end{gathered}
$$

and $\psi_{1}(\cdot)$ is the trigamma function-i.e., $\psi_{1}(x)=\frac{d^{2}}{d x^{2}} \ln \Gamma(x)$.

Remark 4. Asymptotic confidence intervals of the parameters of the $\alpha$-GlogM distribution can be obtained by using observed information matrix $\mathbf{J}(\Theta)$ by assuming the $M L$ estimators has approximately a $N_{3}\left(\Theta, \mathbf{I}(\Theta)^{-1}\right)$ distribution where $\mathbf{I}(\Theta)$ is expected information matrix. It should be noted that expected information matrix $\mathbf{I}(\Theta)$ for $\Theta$ cannot be obtained explicitly; therefore, matrix $\mathbf{J}(\Theta)$ evaluated at $\hat{\Theta}$ can be used in practice. See Iriarte et al. [34] in the context of observed information matrix for parameters of the SGR distribution.

Remark 5. To find ML estimates of the parameters $\alpha, \beta$, and $\sigma$, which the $\ln L$ function in (6) attains its maximum, optimization tools "fminunc" or "fminsearch" whcih are available in software MATLAB2015b can be used. However, finding the maximum value of the $\ln L$ function may have some computational difficulties. To alleviate this problem, I recommend two approaches: (i) using a population-based method such as the genetic algorithm, particle swarm, simulated annealing that these optimization tools are available in MATLAB2015b; (ii) considering the reparameterization $\lambda=\frac{\sigma^{1 / \beta}}{2}$ so that pdf of the $\alpha-G \log M$ distribution is rewritten as

$$
f_{T}(t ; \alpha, \beta, \lambda)=\frac{\alpha}{\lambda^{\alpha \beta} \sqrt{\pi}} \Gamma(\alpha \beta+0.5) t^{\alpha-1} G\left(t^{-\frac{1}{\beta}}, \alpha \beta+0.5, \lambda\right),
$$

where $\alpha$ and $\beta$ are shape parameters and $\lambda$ is the scale parameter.

\subsection{MoM Estimation}

The MoM estimators of the parameters $\alpha, \beta$, and $\sigma$ can be obtained by equating the first three theoretical moments to the corresponding sample moments as given below:

$$
\mathbb{E}[T]=\frac{\alpha \sigma}{\sqrt{\pi} 2^{\beta}(\alpha+1)} \Gamma(0.5-\beta)=T_{1} ; \quad \beta<0.5,
$$




$$
\mathbb{E}\left[T^{2}\right]=\frac{\alpha \sigma^{2}}{\sqrt{\pi} 2^{2 \beta}(\alpha+2)} \Gamma(0.5-2 \beta)=T_{2} ; \quad \beta<0.25
$$

and

$$
\mathbb{E}\left[T^{3}\right]=\frac{\alpha \sigma^{3}}{\sqrt{\pi} 2^{3 \beta}(\alpha+3)} \Gamma(0.5-3 \beta)=T_{3} ; \quad \beta<0.1 \overline{6} .
$$

Here, $T_{1}=(1 / n) \sum_{i=1}^{n} t_{i}, T_{2}=(1 / n) \sum_{i=1}^{n} t_{i}^{2}$ and $T_{3}=(1 / n) \sum_{i=1}^{n} t_{i}^{3}$. From Equation (7), the MoM estimator of the $\sigma$, i.e., $\hat{\sigma}_{M o M}$, is

$$
\hat{\sigma}_{M o M}=\frac{\sqrt{\pi} 2^{\beta}(\alpha+1)}{\alpha \Gamma(0.5-\beta)} T_{1} .
$$

After incorparating the $\hat{\sigma}_{M o M}$ into Equations (8) and (9), then equating them to zore, the equations

$$
\begin{gathered}
\sqrt{\pi} \frac{(\alpha+1)^{2}}{\alpha(\alpha+2)} \frac{\Gamma(0.5-2 \beta)}{\Gamma^{2}(0.5-\beta)} T_{1}^{2}-T_{2}=0, \\
\sqrt{\pi} \frac{(\alpha+1)^{3}}{\alpha^{2}(\alpha+3)} \frac{\Gamma(0.5-3 \beta)}{\Gamma^{3}(0.5-\beta)} T_{1}^{3}-T_{3}=0
\end{gathered}
$$

are obtained.

The MoM estimates of $\alpha$ and $\beta$, i.e., values of the $\hat{\alpha}_{M o M}$ and $\hat{\beta}_{M o M}$, are obtained by solving the system of equations in (11) simultaneously. Here, function "fsolve", available in MATLAB2015b, can be used to solve them. See Olmos et al.'s work [15] for an example in the context of MoM estimation of the parameters of an SGHN distribution.

\subsection{Monte-Carlo Simulation}

In this subsection, performances of the ML and MoM estimators of the $\alpha, \beta$, and $\sigma$ parameters are compared via the Monte-Carlo simulation study. Simulation scenarios

\begin{tabular}{ccccccccc}
\hline Scenario & $\alpha$ & $\boldsymbol{\beta}$ & $\boldsymbol{\sigma}$ & $\mathbb{E}[\boldsymbol{T}]$ & $\mathbb{V}[\boldsymbol{T}]$ & $\sqrt{\boldsymbol{\beta}_{\mathbf{1}}}$ & $\boldsymbol{\beta}_{\mathbf{2}}$ \\
\hline I & 1.8 & 0.10 & 1.0 & 0.7506 & 0.1325 & 1.3991 & 16.7429 \\
II & 3.5 & 0.05 & 2.5 & 2.0856 & 0.2945 & -0.1339 & 4.1345 \\
III & 1.2 & 0.01 & 1.2 & 0.6631 & 0.1148 & -0.1489 & 1.8828 \\
IV & 0.9 & 0.10 & 5.0 & 2.7655 & 3.7250 & 1.1570 & 10.1785 \\
\hline
\end{tabular}

are considered. Values of the $\mathbb{E}[T], \mathbb{V}[T], \sqrt{\beta_{1}}$ and $\beta_{2}$ are calculated by using the Proposition 9 via MATHEMATICA.

All the simulations were conducted for $\lfloor 100,000 / n\rfloor$ Monte-Carlo runs, where $\lfloor\cdot\rfloor$ denotes the integer value function via MATLAB2015b. Here, sample size, $n$, was considered as 20(small), 50(moderate), 100 and 200(large). For each generated sample, the ML estimates of the $\alpha, \beta$, and $\sigma$ parameters were obtained by using the optimization tool "fminunc", i.e., unconstrained minimization function, and the corresponding MoM estimates were computed via the "fsolve" function, both of which are available in the MATLAB2015b. Then, simulated bias, variance, and MSE values for the ML and MoM estimators of the parameters $\alpha, \beta$, and $\sigma$ were computed for each generated sample. The results of the Monte-Carlo simulation study are reported in Table 1.

It can be seen from Table 1 that the simulated bias and variance values for each parameter $\alpha, \beta$, and $\sigma$, and therefore the MSE values of them are small for the large sample sizes for all simulation scenarios. However, the ML method gives lower MSE values than MoM.

Concerning the small sample size, in some cases, the MoM method produces lower MSE values than the ML. It should also be noted that if the sample size increases, then the MSE values for each parameter decrease, as expected. 
Table 1. The simulated bias, variance, and MSE values of the ML and MoM estimators.

\begin{tabular}{|c|c|c|c|c|c|c|c|}
\hline \multirow[t]{2}{*}{$\mathbf{n}$} & & \multicolumn{6}{|c|}{ Scenario-I } \\
\hline & & Bias & Variance & MSE & Bias & Variance & MSE \\
\hline \multirow{3}{*}{20} & $\hat{\alpha}$ & -0.13153 & 0.19941 & 0.21651 & 0.13275 & 0.31909 & 0.33671 \\
\hline & $\hat{\beta}$ & 0.00139 & 0.00192 & 0.00192 & 0.03010 & 0.00077 & 0.00168 \\
\hline & $\hat{\sigma}$ & 0.01034 & 0.01652 & 0.01663 & -0.10730 & 0.02837 & 0.03988 \\
\hline \multirow{3}{*}{50} & $\hat{\alpha}$ & -0.11841 & 0.16049 & 0.17443 & 0.12084 & 0.10649 & 0.12104 \\
\hline & $\hat{\beta}$ & -0.00167 & 0.00080 & 0.00080 & 0.01888 & 0.00042 & 0.00078 \\
\hline & $\hat{\sigma}$ & 0.00215 & 0.00738 & 0.00738 & -0.08608 & 0.00940 & 0.01680 \\
\hline \multirow{3}{*}{100} & $\hat{\alpha}$ & -0.03556 & 0.05736 & 0.05857 & 0.13539 & 0.04900 & 0.06729 \\
\hline & $\hat{\beta}$ & -0.00017 & 0.00042 & 0.00042 & 0.01485 & 0.00034 & 0.00056 \\
\hline & $\hat{\sigma}$ & -0.00157 & 0.00352 & 0.00352 & -0.06626 & 0.00394 & 0.00833 \\
\hline \multirow{3}{*}{200} & $\hat{\alpha}$ & -0.02077 & 0.02844 & 0.02882 & 0.11269 & 0.02589 & 0.03854 \\
\hline & $\hat{\beta}$ & -0.00133 & 0.00020 & 0.00020 & 0.01107 & 0.00024 & 0.00036 \\
\hline & $\hat{\sigma}$ & 0.00140 & 0.00167 & 0.00167 & -0.04923 & 0.00192 & 0.00434 \\
\hline \multirow{3}{*}{$\mathbf{n}$} & & \multicolumn{6}{|c|}{ Scenario-II } \\
\hline & & \multicolumn{3}{|c|}{ ML } & \multicolumn{3}{|c|}{ MoM } \\
\hline & & Bias & Variance & MSE & Bias & Variance & MSE \\
\hline \multirow{3}{*}{20} & $\hat{\alpha}$ & -0.51300 & 1.55340 & 1.81657 & -0.41089 & 1.26007 & 1.42368 \\
\hline & $\hat{\beta}$ & -0.00017 & 0.00066 & 0.00066 & 0.00789 & 0.00059 & 0.00064 \\
\hline & $\hat{\sigma}$ & 0.02281 & 0.03283 & 0.03321 & -0.01732 & 0.03025 & 0.03042 \\
\hline \multirow{3}{*}{50} & $\hat{\alpha}$ & -0.16020 & 0.50873 & 0.53414 & -0.08283 & 0.47270 & 0.47932 \\
\hline & $\hat{\beta}$ & -0.00129 & 0.00023 & 0.00023 & 0.00450 & 0.00036 & 0.00038 \\
\hline & $\hat{\sigma}$ & 0.00035 & 0.01267 & 0.01267 & -0.03229 & 0.01652 & 0.01756 \\
\hline \multirow{3}{*}{100} & $\hat{\alpha}$ & -0.10093 & 0.24034 & 0.25029 & -0.06002 & 0.29182 & 0.29513 \\
\hline & $\hat{\beta}$ & -0.00134 & 0.00011 & 0.00011 & 0.00155 & 0.00017 & 0.00017 \\
\hline & $\hat{\sigma}$ & 0.00443 & 0.00522 & 0.00524 & -0.01462 & 0.00873 & 0.00893 \\
\hline \multirow{3}{*}{200} & $\hat{\alpha}$ & -0.06286 & 0.09844 & 0.10219 & -0.03714 & 0.11643 & 0.11758 \\
\hline & $\hat{\beta}$ & -0.00109 & 0.00005 & 0.00005 & 0.00078 & 0.00007 & 0.00007 \\
\hline & $\hat{\sigma}$ & 0.00295 & 0.00230 & 0.00230 & -0.00865 & 0.00396 & 0.00403 \\
\hline & & & & Scer & io-III & & \\
\hline $\mathbf{n}$ & & & ML & & & MoM & \\
\hline & & Bias & Variance & MSE & Bias & Variance & MSE \\
\hline & $\hat{\alpha}$ & -0.13570 & 0.10631 & 0.12462 & -0.26612 & 0.19742 & 0.26812 \\
\hline 20 & $\hat{\beta}$ & -0.03644 & 0.00072 & 0.00205 & -0.02095 & 0.00099 & 0.00143 \\
\hline & $\hat{\sigma}$ & 0.15882 & 0.01057 & 0.03579 & 0.12531 & 0.01390 & 0.02959 \\
\hline & $\hat{\alpha}$ & -0.09049 & 0.04164 & 0.04981 & -0.09361 & 0.04758 & 0.05631 \\
\hline 50 & $\hat{\beta}$ & -0.02101 & 0.00027 & 0.00071 & -0.01392 & 0.00070 & 0.00089 \\
\hline & $\hat{\sigma}$ & 0.09206 & 0.00414 & 0.01262 & 0.07876 & 0.00807 & 0.01427 \\
\hline & $\hat{\alpha}$ & -0.04574 & 0.01675 & 0.01882 & -0.05437 & 0.01966 & 0.02260 \\
\hline 100 & $\hat{\beta}$ & -0.01440 & 0.00009 & 0.00030 & -0.01046 & 0.00051 & 0.00062 \\
\hline & $\hat{\sigma}$ & 0.05926 & 0.00167 & 0.00518 & 0.05553 & 0.00516 & 0.00824 \\
\hline & $\hat{\alpha}$ & -0.03068 & 0.00843 & 0.00936 & -0.04136 & 0.01091 & 0.01260 \\
\hline 200 & $\hat{\beta}$ & -0.01024 & 0.00002 & 0.00013 & -0.00801 & 0.00046 & 0.00052 \\
\hline & $\hat{\sigma}$ & 0.04120 & 0.00052 & 0.00222 & 0.04334 & 0.00323 & 0.00510 \\
\hline & & & & Scer & io-IV & & \\
\hline $\mathbf{n}$ & & & ML & & & MoM & \\
\hline & & Bias & Variance & MSE & Bias & Variance & MSE \\
\hline & $\hat{\alpha}$ & -0.11095 & 0.08495 & 0.09718 & -0.01709 & 0.06358 & 0.06381 \\
\hline 20 & $\hat{\beta}$ & 0.02055 & 0.00438 & 0.00480 & 0.04348 & 0.00140 & 0.00329 \\
\hline & $\hat{\sigma}$ & -0.00458 & 0.89955 & 0.89867 & -0.48331 & 0.77694 & 1.00975 \\
\hline & $\hat{\alpha}$ & -0.02869 & 0.02844 & 0.02918 & 0.07019 & 0.02352 & 0.02838 \\
\hline 50 & $\hat{\beta}$ & -0.01916 & 0.00137 & 0.00173 & 0.01161 & 0.00041 & 0.00055 \\
\hline & $\hat{\sigma}$ & 0.01344 & 0.42467 & 0.42354 & -0.60497 & 0.43470 & 0.79936 \\
\hline & $\hat{\alpha}$ & -0.02454 & 0.01245 & 0.01302 & 0.05199 & 0.01208 & 0.01476 \\
\hline 100 & $\hat{\beta}$ & -0.01551 & 0.00065 & 0.00089 & 0.00791 & 0.00037 & 0.00043 \\
\hline & $\hat{\sigma}$ & 0.02554 & 0.19268 & 0.19285 & -0.44551 & 0.14778 & 0.34589 \\
\hline & $\hat{\alpha}$ & -0.00952 & 0.00617 & 0.00625 & 0.04917 & 0.00687 & 0.00928 \\
\hline 200 & $\hat{\beta}$ & -0.01128 & 0.00026 & 0.00038 & 0.00507 & 0.00028 & 0.00030 \\
\hline & $\hat{\sigma}$ & 0.00976 & 0.07829 & 0.07823 & -0.32986 & 0.07196 & 0.18063 \\
\hline
\end{tabular}


Covarage propability (CP) values based on the ML estimators are given in Table 2. Here, inverse of the observed information matrix rather than inverse of the expected information matrix is utilized, and preassumed values for $\mathrm{CP}$ are taken to be $95 \%$, e.g., $\hat{\alpha}-1.96 \times \mathbf{J}^{-1}(\hat{\Theta})_{11} \leq \alpha \leq \hat{\alpha}+1.96 \times \mathbf{J}^{-1}(\hat{\Theta})_{11}$ for $\alpha$; see Remark 4 .

Table 2. The simulated covarage probability values of the ML estimators.

\begin{tabular}{cccccc}
\hline \multicolumn{1}{c}{$\mathbf{n}$} & & Scenario-I & Scenario-II & Scenario-III & Scenario-IV \\
\hline \multirow{2}{*}{20} & $\hat{\alpha}_{M L}$ & 0.96 & 0.97 & 0.96 & 0.93 \\
& $\hat{\beta}_{M L}$ & 0.89 & 0.91 & 0.87 & 0.96 \\
& $\hat{\sigma}_{M L}$ & 0.90 & 0.93 & 0.90 & 0.87 \\
\hline \multirow{2}{*}{50} & $\hat{\alpha}_{M L}$ & 0.95 & 0.95 & 0.95 & 0.94 \\
& $\hat{\beta}_{M L}$ & 0.94 & 0.92 & 0.88 & 0.95 \\
& $\hat{\sigma}_{M L}$ & 0.99 & 0.90 & 0.98 & 0.98 \\
\hline \multirow{2}{*}{100} & $\hat{\alpha}_{M L}$ & 0.96 & 0.95 & 0.96 & 0.95 \\
& $\hat{\beta}_{M L}$ & 0.93 & 0.95 & 0.89 & 0.91 \\
\hline \multirow{2}{*}{200} & $\hat{\sigma}_{M L}$ & 0.99 & 0.93 & 0.99 & 0.94 \\
& $\hat{\alpha}_{M L}$ & 0.94 & 0.96 & 0.90 & 0.97 \\
& $\hat{\beta}_{M L}$ & 0.95 & 0.95 & 0.98 & 0.94 \\
\hline
\end{tabular}

It is clear from Table 2 that the simulated CP values get closer to the preassumed value of $95 \%$ when the sample size increases. Additionally, the CP results show that the $\alpha, \beta$ and $\sigma$ parameters are not under- or overestimated by corresponding ML estimators when the $n$ becomes larger.

\section{Applications}

As stated in the introduction, many authors have tried to find new statistical distributions to model environmental events more accurately. For example, Bakouch et al. [5] proposed to use BE2 distribution to model the rainfall data and Gómez et al. [7] introduced the SG distribution to model the snow accumulation data. In this section, two environmental data sets from Bakouch et al. [5] and Gómez et al. [7] are modeled by using the $\alpha$-GlogM distribution. The ML and MoM methodologies are used to obtain the estimates of the $\alpha, \beta$ and $\sigma$ parameters of the $\alpha$-GlogM distribution. The ML and MoM estimates of $\alpha, \beta$ and $\sigma$ are obtained as in Sections 4.1 and 4.2. Modeling performances of the $\alpha$-GlogM distribution and its rivals are also compared. The comparisons are carried out using well-known information criteria (IC), e.g., $\ln L$, Akaike information criterion (AIC), and corrected AIC (AICc). Additionally, goodness-of-fit statistics, e.g., Anderson-Darling (AD), Kolmogorov-Smirnov (KS), coefficient of determination $\left(R^{2}\right)$, and root mean squared error (RMSE) methods are used in the comparisons. The formulas for them are

$\begin{array}{cc}\mathrm{AIC}= & -2 \ln L+2 k \\ \mathrm{AIC}= & \mathrm{AIC}+(2 k(k+1)) /(n-k-1) \\ \mathrm{RMSE}= & {\left[\frac{1}{n} \sum_{i=1}^{n}\left(F\left(x_{(i)} ; \hat{\Theta}\right)-\frac{i}{n+1}\right)^{2}\right]^{1 / 2}} \\ R^{2}= & 1-\frac{\sum_{i=1}^{n}\left(F\left(x_{(i)} ; \hat{\Theta}\right)-\frac{i}{n+1}\right)^{2}}{\sum_{i=1}^{n}\left(F\left(x_{(i)} ; \hat{\Theta}\right)-\bar{F}\left(x_{(i)} ; \hat{\Theta}\right)\right)^{2}}, 0<R^{2}<1 \\ \mathrm{KS}= & \max \left|F\left(x_{(i)} ; \hat{\Theta}\right)-\frac{i}{n+1}\right| \\ \mathrm{AD}= & -n-\sum_{i=1}^{n}\left(\frac{2 i-1}{n}\right)\left[\ln \left(F\left(x_{(i)} ; \hat{\Theta}\right)\right)+\ln \left(1-F\left(x_{(n+1-i)} ; \hat{\Theta}\right)\right)\right]\end{array}$

where $\bar{F}\left(x_{(i)} ; \hat{\Theta}\right)=\frac{1}{n} \sum_{i=1}^{n} F\left(x_{(i)} ; \hat{\Theta}\right)$. Here, $k, n, \hat{\Theta}$ and $x_{(\cdot)}$ denote number of parameters, sample size, estimated parameter vector and ordered observations in ascending order, respectively. Note that smaller values of the AIC, AICc, RMSE, AD and KS and a higher value of $\ln L$ and $R^{2}$ mean better modeling performance. 
Remark 6. The $\alpha-G \log M$ distribution has two shape parameters along with a scale parameter. However, the BE2 and SG distributions only have one shape parameter with a scale parameter. Therefore, the modeling performance of the $\alpha$-GlogM distribution should be compared with the more competitive model. For this purpose, the SGR distribution proposed by Iriarte et al. [34], having two shape parameters and one scale parameter, is included in the comparisons to make the study complete.

\subsection{Application-I}

In this subsection, total monthly rainfall data from Bakouch et al. [5] are considered to show the modeling capability of the $\alpha-\operatorname{Glog} \mathrm{M}(\alpha, \beta, \sigma)$ distribution. The data set contains 53 observations from Sao Carlos, Brazil, between the years 1960 to 2014 for April. The total monthly rainfall data set is given in Table 3 .

Table 3. The total monthly rainfall data $(n=53)$.

\begin{tabular}{lllllllllll}
\hline 59.00 & 102.20 & 17.30 & 23.00 & 50.60 & 27.00 & 203.00 & 40.90 & 53.00 & 177.40 & 94.60 \\
129.40 & 76.00 & 93.20 & 22.80 & 98.80 & 77.70 & 204.20 & 16.90 & 55.10 & 103.90 & 34.90 \\
39.70 & 137.70 & 104.20 & 117.60 & 17.10 & 120.80 & 164.90 & 50.20 & 172.80 & 58.50 & 112.40 \\
24.50 & 32.80 & 64.00 & 72.10 & 139.30 & 0.50 & 70.90 & 0.80 & 82.70 & 108.60 & 32.30 \\
13.60 & 25.70 & 135.80 & 136.80 & 89.70 & 139.20 & 102.80 & 97.30 & 60.60 & & \\
\hline
\end{tabular}

Note that Bakouch et al. [5] considered not only the BE2 distribution but also the Weibull, Gamma, Log-normal, Gumbel, and generalized exponential distributions for modeling the data given in Table 3 . They showed that the BE2 distribution is preferable over these distributions when the AIC, AICc, and KS criteria are taken into account. Therefore, the Weibull, Gamma, Log-normal, Gumbel, and generalized exponential distributions are not included to comparisons for sake of brevity.

The parameter estimates of the $\alpha$-GlogM, BE2, and SGR distributions and corresponding IC values and goodness-of-fit statistics for them are given in Table 4.

Table 4. Results for the total monthly rainfall data.

\begin{tabular}{|c|c|c|c|c|c|c|c|c|c|}
\hline \multicolumn{10}{|c|}{$\alpha-\mathrm{G} \log M$ distribution } \\
\hline$\hat{\alpha}_{M L}$ & $\hat{\beta}_{M L}$ & $\hat{\sigma}_{M L}$ & $\ln L$ & AIC & $\mathrm{AICc}$ & $\mathrm{AD}$ & KS & RMSE & $R^{2}$ \\
\hline 0.9390 & 0.0931 & 145.6986 & -279.4682 & 564.9364 & 565.4262 & 0.2563 & 0.0638 & 0.0201 & 0.9950 \\
\hline$\hat{\alpha}_{M o M}$ & $\hat{\beta}_{M o M}$ & $\hat{\sigma}_{M o M}$ & $\ln L$ & AIC & $\mathrm{AICc}$ & $\mathrm{AD}$ & KS & RMSE & $R^{2}$ \\
\hline 0.9407 & 0.0726 & 150.0158 & - & - & - & 0.3088 & 0.0622 & 0.0223 & 0.9938 \\
\hline \multicolumn{10}{|c|}{ BE2 distribution } \\
\hline$\hat{\theta}_{M L}$ & $\hat{\lambda}_{M L}$ & & $\ln L$ & AIC & $\mathrm{AICc}$ & $\mathrm{AD}$ & KS & RMSE & $R^{2}$ \\
\hline 0.9100 & 0.0227 & & -281.2113 & 566.4226 & 566.6626 & 0.5986 & 0.1002 & 0.0407 & 0.9796 \\
\hline \multicolumn{10}{|c|}{ SGR distribution } \\
\hline$\hat{\theta}_{M L}$ & $\hat{\alpha}_{M L}$ & $\hat{q}_{M L}$ & $\ln L$ & $\mathrm{AIC}$ & $\mathrm{AICc}$ & $\mathrm{AD}$ & KS & RMSE & $R^{2}$ \\
\hline 0.0001 & -0.4161 & 22.4821 & -279.9818 & 565.9637 & 566.4534 & 0.4296 & 0.0888 & 0.0334 & 0.9865 \\
\hline
\end{tabular}

It can be seen from Table 4 that the AIC, RMSE, AD and KS values for the $\alpha$-GlogM distribution are smaller, and that $\ln L$ and $R^{2}$ are greater than the corresponding values for the BE2 and SGR distributions. These values show that the $\alpha$-GlogM distribution exhibits a better modeling performance than the BE2 and SGR distributions. Therefore, the $\alpha$-GlogM distribution can be considered as an alternative to the BE2 and SGR distributions. The fitting performance of the $\alpha$-GlogM distribution and surface plot of the $\ln L$ function are also illustrated in Figure 3. 


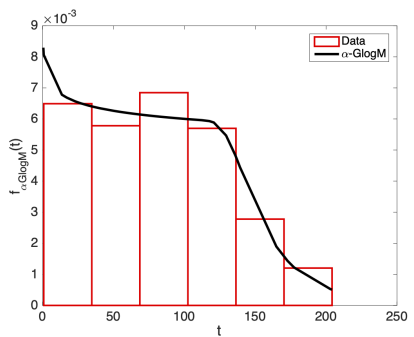

(a) pdf

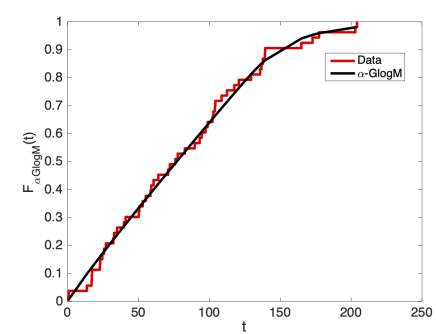

(b) cdf

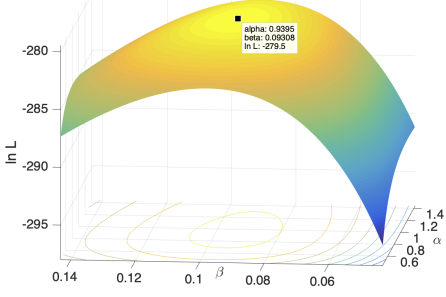

(c) $\ln L \quad\left(\hat{\sigma}_{M L}=145.6986\right)$

Figure 3. Fitting plots and surface plot of the $\ln L$ for the total monthly rainfall data.

\subsection{Application-II}

In this subsection, the data set involves 63 observations for the snow accumulation in inches for the Raleigh-Durham airport in North Carolina, modeled by using the $\alpha$-GlogM distribution-see Gómez et al. [7] for further details. The snow accumulation data are given in Table 5 .

Table 5. The snow accumulation data $(n=63)$.

\begin{tabular}{|c|c|c|c|c|c|c|c|c|c|c|c|c|c|c|c|c|}
\hline 1.0 & 2.5 & 1.2 & 1.2 & 4.1 & 9.0 & 3.0 & 1.0 & 1.4 & 2.0 & 3.0 & 1.7 & 1.2 & 1.2 & 1.1 & 1.5 & 5.0 \\
\hline 1.6 & 2.0 & 0.1 & 0.4 & 0.8 & 3.7 & 1.3 & 3.8 & 0.1 & 0.1 & 0.2 & 2.0 & 7.6 & 0.1 & 1.8 & 0.5 & 0.5 \\
\hline 0.5 & 1.1 & 1.4 & 1.0 & 1.0 & 0.7 & 5.7 & 0.4 & 0.3 & 1.8 & 0.4 & 1.0 & 1.2 & 2.6 & 1.0 & 5.0 & 1.7 \\
\hline 2.4 & 0.1 & 0.5 & 7.1 & 0.2 & 0.7 & 0.1 & 2.7 & 2.9 & 0.4 & 2.0 & 20.3 & & & & & \\
\hline
\end{tabular}

Note that Gómez et al. [7] considered not only the SG distribution, but also the Gumbel and slash distributions for modeling the data given in Table 5. They showed that the SG distribution is preferable over the Gumbel and slash distributions when the AIC and modified AD criteria are taken into account. Therefore, the Gumbel and slash distributions are not included for comparisons for sake of brevity.

The parameter estimates of the $\alpha$-GlogM, SG and SGR distributions and corresponding IC values and goodness-of-fit statistics for them are given in Table 6.

Table 6. Results for the snow accumulation data.

\begin{tabular}{|c|c|c|c|c|c|c|c|c|}
\hline \multicolumn{9}{|c|}{$\alpha-G \log M$ distribution } \\
\hline$\hat{\alpha}_{M L}$ & $\hat{\beta}_{M L}$ & $\hat{\sigma}_{M L}$ & $\ln L$ & AIC & $\mathrm{AD}$ & $\mathrm{KS}$ & RMSE & $R^{2}$ \\
\hline 0.9706 & 0.3332 & 2.0709 & -107.2595 & 220.5190 & 0.2999 & 0.0790 & 0.0247 & 0.9929 \\
\hline$\hat{\alpha}_{M o M}$ & $\hat{\beta}_{M o M}$ & $\hat{\sigma}_{M o M}$ & $\ln L$ & AIC & $\mathrm{AD}$ & KS & RMSE & $R^{2}$ \\
\hline 1.9497 & 0.1623 & 2.4131 & - & - & 15.4246 & 0.3175 & 0.1754 & 0.7543 \\
\hline \multicolumn{9}{|c|}{ SG distribution } \\
\hline$\hat{\mu}_{M L}$ & $\hat{\sigma}_{M L}$ & $\hat{q}_{M L}$ & $\ln L$ & $A I C$ & $\mathrm{AD}$ & KS & RMSE & $R^{2}$ \\
\hline 0.876 & 0.557 & 1.637 & -116.496 & 238.992 & 1.0556 & 0.1105 & 0.043 & 0.9783 \\
\hline \multicolumn{9}{|c|}{ SGR distribution } \\
\hline$\hat{\theta}_{M L}$ & $\hat{\alpha}_{M L}$ & $\hat{q}_{M L}$ & $\ln L$ & AIC & $\mathrm{AD}$ & KS & RMSE & $R^{2}$ \\
\hline 0.4423 & -0.4779 & 1.5611 & -107.3915 & 220.7830 & 0.3131 & 0.0891 & 0.0253 & 0.9925 \\
\hline
\end{tabular}

It can be seen from Table 6 that the AIC, RMSE, AD and KS values, obtained by using the ML estimates, of the $\alpha$-GlogM distribution are smaller, and $\ln L$ and $R^{2}$ are greater than the corresponding values for the SG and SGR distributions. Hence, the $\alpha-G \log M$ distribution exhibits a better modeling performance than the SG and SGR distributions. The fitting performance of the $\alpha$-GlogM distribution and surface plot of the $\ln L$ function are also illustrated in Figure 4. 


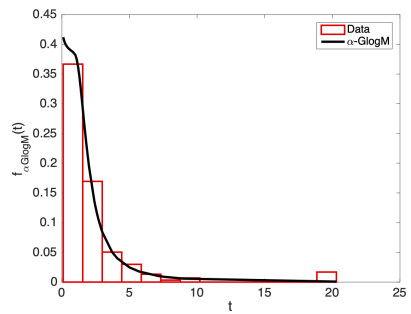

(a) pdf

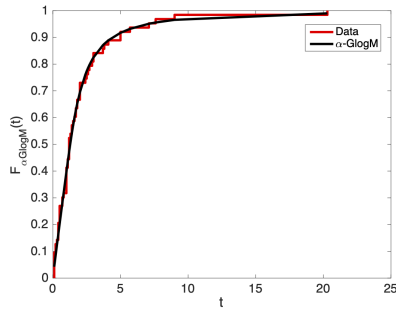

(b) $\mathrm{cdf}$

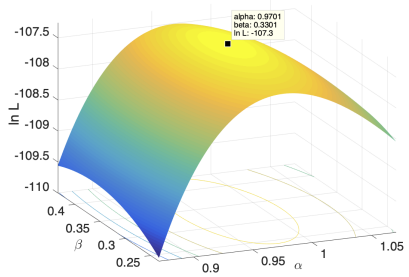

(c) $\ln L \quad\left(\hat{\sigma}_{M L}=2.0709\right)$

Figure 4. Fitting plots and surface plot of the $\ln L$ for the snow accumulation data.

\section{Conclusions}

In this study, the $\alpha$-GlogM distribution is introduced, and some statistical properties of it are derived. Then, the $\alpha$-GlogM distribution is used to model the environmental data sets from the different environmental events. Additionally, the modeling capability of the $\alpha$-GlogM and its rivals, e.g., the BE2, SG, and SGR, are compared by using the well-known IC and goodness-of-fit statistics. Results show that the $\alpha$-GlogM distribution is preferable over the BE2, SG, and SGR distributions for modeling these data sets. It can be concluded that the $\alpha-\mathrm{G} \log \mathrm{M}$ distribution can be considered as an alternative to the popular distributions for modeling the environmental data.

Funding: This research received no external funding.

Acknowledgments: The author would like to thank the reviewers for their valuable comments and suggestions which improved the paper.

Conflicts of Interest: The author declare no conflict of interest.

\section{References}

1. Gómez, H.W.; Venegas, O.; Bolfarine, H. Skew-symmetric distributions generated by the distribution function of the normal distribution. Environmetrics 2007, 18, 395-407. [CrossRef]

2. Leiva, V.; Barros, M.; Paula, G.A.; Sanhueza, A. Generalized Birnbaum-Saunders distributions applied to air pollutant concentration. Environmetrics 2007, 19, 235-249. [CrossRef]

3. Nadarajah, S. A truncated inverted beta distribution with application to air pollution data. Stoch. Environ. Res. Risk. Assess. 2008, 22, 285-289. [CrossRef]

4. Martínez-Flórez, G.; Bolfarine, H.; Gómez, H.W. The log-power-normal distribution with application to air pollution. Environmetrics 2014, 25, 44-56. [CrossRef]

5. Bakouch, H.S.; Dey, S.; Ramos, P.L.; Louzada, F. Binomial-exponential 2 distribution: Different estimation methods with weather applications. TEMA (São Carlos) 2017, 18, 233-251. [CrossRef]

6. Asgharzadeh, A.; Bakouch, H.S.; Habibi, M. A generalized binomial-exponential 2 distribution: Modeling and applications to hydrologic events. J. Appl. Stat. 2017, 44, 2368-2387. [CrossRef]

7. Gómez, Y.M.; Bolfarine, H.; Gomez, H.W. Gumbel distribution with heavy tails and applications to environmental data. Math Comput. Simul. 2019, 157, 115-129. [CrossRef]

8. Bahti, D.; Ravi, S. On generalized log-Moyal distribution: A new heavy-tailed size distribution. Insur. Math. Econom. 2018, 79, 247-259. [CrossRef]

9. Lee, C.; Famoye, F.; Alzaatreh, A.Y. Methods for generation families of univariate continuous distributions in the recent decades. Wiley Interdiscip. Rev. Comput. Stat. 2013, 5, 219-238. [CrossRef]

10. Moyal, J.E. Theory of ionization fluctuations. Lond. Edinb. Dublin Philos. Mag. J. Sci. 1955, 46, 263-280. [CrossRef]

11. Walck, C. Hand-Book on Statistical Distributions for Experimentalists; Particle Physics Group, Fysikum, University of Stockholm: Stockholm, Sweden, 2007.

12. Cordeiro, G.M.; Nobre, J.S.; Pescim, R.R.; Ortega, E.M.M. The beta Moyal: A useful skew distribution. Int. J. Res. Rev. Appl. Sci. 2012, 10, 171-192.

13. Genc, A.A.; Korkmaz, M.C.; Kus, C. The Beta Moyal-Slash distribution. J. Selçuk Univ. Nat. Appl. Sci. 2014, 3, 88-104.

14. Olmos, N.M.; Varela, H.; Bolfarine, H.; Gómez, H.W. An extension of the half-normal distribution. Stat. Pap. 2012, 53, 875-886. [CrossRef]

15. Olmos, N.M.; Varela, H.; Bolfarine, H.; Gómez, H.W. An extension of the generalized half-normal distribution. Stat. Pap. 2014, 55, 967-981. [CrossRef] 
16. Cooray, K.; Ananda, M.A.M. A generalization of the half-normal distribution with applications to lifetime data. Commun. Stat. Theory Methods 2008 37, 1323-1337. [CrossRef]

17. Andrews, D.D.; Bickel, P.J.; Hampel, F.Y.; Huber, P.J.; Rogers, W.H.; Tukey, J.W. Robust Estimates of Location: Survey and Advances; Princeton University Press: Princeton, NJ, USA, 1972.

18. Rogers,W.H.; Tukey, J.W. Understanding some long-tailed symmetrical distributions. Stat. Neerl. 1972, 26, 211-226. [CrossRef]

19. Mosteller, F.; Tukey, J.W. Data Analysis and Regression; Addison-Wesley: Reding, MA, USA, 1977.

20. Jones, M.C. On univariate slash distributions, continuous and discrete. Ann. Inst. Stat. Math. 2020, 72, 645-657. [CrossRef]

21. Gómez, H.W.; Quintana, F.A.; Torres, J. A new family of slash-distributions with elliptical contours. Stat. Probab. Lett. 2007, 77, 717-725. [CrossRef]

22. Genc, A.I. A generalization of the univariate slash by a scale-mixtured exponential power distribution. Commun. Stat. Simul. Comput. 2007, 36, 937-947. [CrossRef]

23. Punathumparambath, B. A New Family of Skewed Slash Distributions Generated by the Cauchy Kernel. Commun. Stat. Theory Methods 2013, 42, 2351-2361. [CrossRef]

24. Astorga, J.M.; Gomez, H.W.; Bolfarine, H. Slashed generalized exponential distribution. Commun. Stat. Theory Methods 2017, 46, 2091-2102. [CrossRef]

25. Korkmaz, M.C. A generalized skew slash distribution via gamma-normal distribution. Commun. Stat. Simul. Comput. 2017, 46, 1647-1660. [CrossRef]

26. Arslan, O. An alternative multivariate skew-slash distribution. Stat. Probab. Lett. 2008 78, 2756-2761. [CrossRef]

27. Arslan, O.; Genc, A.I. A generalization of the multivariate slash distribution. J. Stat. Plan. Inference 2009, 139, 1164-1170. [CrossRef]

28. Reyes, J.; Gómez, H.W.; Bolfarine, H. Modified slash distribution. Statistics 2013, 47, 929-941. [CrossRef]

29. Iriarte, Y.A.; Castillo, N.O.; Bolfarine, J.; Gómez, H.W. Modified slashed-Rayleigh distribution. Commun. Stat. Theory Methods 2018, 47, 3220-3233. [CrossRef]

30. Reyes, J.; Barranco-Chamorro, I.; Gómez, H.W. Generalized modified slash distribution with applications. Commun. Stat. Theory Methods 2020, 49, 2025-2048. [CrossRef]

31. Rojas, M.A.; Bolfarinei, H.; Gómez, H.W. An extension of the slash-elliptical distribution. SORT 2014, 38, $215-230$.

32. Zörnig, P. On generalized slash distributions: Representation by hypergeometric functions. Stats 2019, 2, 371-387. [CrossRef]

33. Arslan, T. Scale-Mixture Extension of Inverse Weibull Distribution, Olomoucian Days of Applied Mathematics 2019; ODAM: Olomouc, Czech Republic, 2019; p. 12.

34. Iriarte, Y.A.; Vilca, F.; Varela, H.; Gómez, H.W. Slashed generalized Rayleigh distribution. Commun. Stat. Theory Methods 2017, 46, 4686-4699. [CrossRef] 